\title{
Namen, Nasen, Narben \\ Zur Physiognomik in Jean Pauls Komet
}

\begin{abstract}
Unsere Seele schreibt mit vierundzwanzig Zeichen der Zeichen (d.h. mit vierundzwanzig Buchstaben der Wörter) an Seelen; die Natur mit Millionen. Sie zwingt uns, an fremde Ichs neben unserem zu glauben, da wir ewig nur Körper sehen - also unsere Seele in fremde Augen, Nasen, Lippen überzutragen. Kurz, durch Physiognomik und Pathognomik beseelen wir erstlich alle Leiber - später alle unorganisierte Körper.
\end{abstract}

(Jean Paul, Über die natürliche Magie der Einbildungskraft) ${ }^{1}$

Die Physiognomik wie die Physiognomik-Kritik spielen im Werk Jean Pauls seit den frühen Satiren eine beträchtliche Rolle. Wie der eingangs zitierte locus classicus aus Über die natürliche Magie der Einbildungskraft (1795) erhellt, ${ }^{2}$ ist Physiognomik für Jean Paul eine Variante, über die Kluft zwischen Körper und Seele wie über die (Un-)Möglichkeit, diese zu überbrücken, nachzudenken. Jean Pauls Interesse an der Physiognomik geht basal auf das Konto seiner dualistischen Anthropologie, die das Verhältnis von Seele und Leib in widerstreitenden Modellen - der Harmonie und der Divergenz - verhandelt. Dass Jean Paul der Physiognomik grundsätzlich zwiespältig gegenübersteht, wird bereits in den frühen Texten Was algemeines über's Physiognomiren und Zur Physiognomik (1780) deutlich, in denen er einerseits begeistert fordert, »den Menschen« zu »bephysiognomiren«, andererseits jedoch bezweifelt, aus den festen Zügen »den Karakter herausphysiognomiren« zu können (II/1, 44) oder in einem einzelnen "Bild eines Menschen" die »Geschichte seines Lebens [...] eingegraben [zu] lesen« (II/1, 86). Der "Fehler aller Physiognomisten«, so Jean Paul im Physiognomischen Postskript über die Nasen der Menschen (1789), sei der Zirkelschluss, dass sie das aus dem Gesicht lesen, "was sie vorher auf eine viel gewissere Weise schon wusten« (II/2, 305). Anders als die Physiognomik

1 Jean Paul: Werke, hg. von Norbert Miller, 10 Bde., München 1959-1985. Jean Pauls Werke werden im Folgenden nach dieser Ausgabe wie folgt zitiert: Abteilung (römische Ziffer)/Band (arabische Ziffer), Seitenzahl (arabische Ziffer), hier I/4, S. 203f.

2 Siehe auch Elsbeth Dangel-Pelloquin: Eigensinnige Geschöpfe. Jean Pauls poetische Geschlechter-Werkstatt, Freiburg i.Br. 1999, S. 30f. 
Johann Caspar Lavaters ${ }^{3}$ betont Jean Paul immer wieder die Arbitrarität nicht nur der willkürlichen, sondern auch der natürlichen Zeichen. Ist der auf die äußere Wahrnehmung von Körpern verwiesene Mensch zum Entziffern gezwungen, bewerkstelligt er diese hermeneutische Aufgabe mittels seiner Phantasie insofern metamorphotisch, als er das eigene Innere in die fremde Physiognomie überträgt. Dergestalt ist Physiognomieren für Jean Paul immer schon Projizieren. Die hier aufscheinende Affinität der Physiognomik zu Ästhetik, Semiotik, Hermeneutik und Poetik erklärt ihr Reflexionspotential für die Literatur. Da sie auch eine `Bildwissenschaft ist,${ }^{4}$ bietet sie Jean Paul nicht zuletzt einen Fond zur Reflexion literarischer Bildlichkeit.

In seinem letzten, Fragment gebliebenen Roman Der Komet oder Nikolaus Marggraf. Eine komische Geschichte, der 1820 bis 1822 in drei Bänden erscheint und dessen Held Nikolaus Marggraf sich zeitweilig u.a. für »Kaspar Lavater den zweiten« $(I / 6,591)$ hält, wuchern physiognomische Lektüren und die Transformation diverser Theoreme der älteren und der Lavater'schen Physiognomik sowie der Phrenologie Galls. Angesichts der persistierenden Indienstnahme von physiognomischen Bildern, Szenen und Narrativen im Werk Jean Pauls ist dabei nach der historischen wie poetologischen Spezifik ihrer Verwendung im Komet zu fragen. Immer wieder wird der Komet als Moderne-Roman gelesen, der ein Bild der "Scheinwelt" der Restauration zeichnet ${ }^{5}$ und dabei die moderne »Finanzökonomie«, die zunehmende Bürokratisierung sowie den »Schimären-Charakter des Subjekts « ${ }^{6}$ literarisch reflektiert. Für seine literarische Diagnose des »Zeitgeistes« (I/6, 673) greift Jean

3 Johann Caspar Lavater: Physiognomische Fragmente zur Beförderung der Menschenkenntniss und Menschenliebe, Bde. I-IV, Faksimiledruck nach der Ausgabe 1775-1778, Zürich 1968-1969. Im Folgenden zitiert als F mit römischer Band- und arabischer Seitenzahl. Vgl. zu Lavaters Semiotik stellvertretend Richard T. Gray: Die Geburt des Genies aus dem Geiste der Aufklärung - Semiotik und Aufklärungsideologie in der Physiognomik Johann Kaspar Lavaters, in: Poetica. Zeitschrift für Sprach- und Literaturwissenschaft 23 (1991), S. 95-138, bes. S. 116.

4 Gemäß Daniela Bohde: Physiognomische Denkfiguren in Kunstgeschichte und visuellen Wissenschaften. Lavater und die Folgen, in: Zeitschrift für Ästhetik und Allgemeine Kunstwissenschaft 56/1 (2011), S. 89-121, hier S. 89.

5 Walter Benjamin: Der eingetunkte Zauberstab. Zu Max Kommerells Jean Pauk, in: ders.: Wahlverwandtschaften. Aufsätze und Reflexionen über deutschsprachige Literatur, ausgewählt und mit einem Nachwort von Jan Philipp Reemtsma, Frankfurt a.M. 2007, S. 125-132, hier S. 130. Vgl. Uwe Schweikert: Jean Pauls »Komet«. Selbstparodie der Kunst, Stuttgart 1971, S. 124-136, bes., S. 127.

6 Werner Nell: Der Ledermann, das Goldkochen und die portable Stadt. Vormoderne Voraussetzungen einer Selbstbeschreibung der Moderne in Jean Pauls Komet, in:Jahrbuch der Jean-Paul-Gesellschaft 48/49 (2013/14), S. 39-56, hier S. 49 und 44. 
Paul in seinem "Archivroman[ ] «7 auch auf die Physiognomik zurück. Sie dient dabei gleichermaßen der Reflexion von Modernisierungsprozessen wie des eigenen Textes, was im Folgenden in Lektüreprotokollen gezeigt wird: Der Romantitel unterlegt dem Text ein sphysiognomisches Programm und unterminiert es zugleich (1). Das führt im Erzählen wie im Erzählten zu intrikaten physiognomischen Szenen, die anhand der Schauplätze Körper (2), Schrift (3) und Bild (4) untersucht werden.

\section{Antlitz des Buchs}

In der Vorrede erklärt Jean Paul den Titel mit der »Ähnlichkeit« von Held und Komet. Durch die gesetzte Ähnlichkeit (vgl. I/6, 568f.) ${ }^{8}$ alludiert er die antike Denkfigur der Analogie von Mikro- und Makrokosmos, die in der hermetischen Vorgeschichte der Physiognomik von Bedeutung ist. ${ }^{9}$ Jean Paul verschaltet im Komet die Magia Naturalis mit der Lavater'schen Physiognomik, die bekanntlich »esoterische [ ] Bestandteile[] enthält. ${ }^{10}$ Lavater geht davon aus, dass "durch das Aeußerliche eines Menschen sein Innres zu erkennen « ist (F I, 13), und setzt so auf eine ungestörte Referentialität, die stabile Bedeutungen garantieren soll. ${ }^{11}$ Auch Jean Paul mangelt es nicht an

7 Oliver Simons: Jean Pauls imaginäre Epistemologie, in: Kultur-Schreiben als romantisches Projekt. Romantische Ethnographie im Spannungsfeld zwischen Imagination und Wissenschaft, hg. von David E. Wellbery, Würzburg 2012, S. 179-191, hier S. 181.

8 Zur Ähnlichkeit vgl. Götz Müller: Mehrfache Kodierung bei Jean Paul, in: ders.: Jean Paul im Kontext. Gesammelte Aufsätze, mit einem Schriftenverzeichnis hg. von Wolfgang Riedel, Würzburg 1996, S. 77-96, hier S. 87 und 94. Zur Episteme des Ähnlichen vgl. Michel Foucault: Die Ordnung der Dinge. Eine Archäologie der Humanwissenschaften, 23. Aufl., Frankfurt a.M. 2015, S. 46-77, 78-82, 269-274.

9 Zur älteren Physiognomik bei Jean Paul vgl. Götz Müller: Jean Pauls Ästhetik und Naturphilosophie, Tübingen 1983, S. 81-86; Gunnar Och: Der Körper als Zeichen. Zur Bedeutung des mimisch-gestischen und physiognomischen Ausdrucks im Werk Jean Pauls, Erlangen 1985, S. 51; Jürgen Brummack: Natürliche Magie, Magnetismus, Alchemie. Über Jean Pauls Komet, in: Hermetik. Literarische Figurationen zwischen Babylon und Cyberspace, hg. von Nicola Kaminski, Heinz J. Drügh und Michael Herrmann, Tübingen 2002, S. 129-160.

10 Annette Graczyk: Lavaters Neubegründung der Physiognomik zwischen Aufklärung, christlicher Religion und Esoterik, in: Aufklärung und Esoterik. Wege in die Moderne, hg. von Monika Neugebauer-Wölk, Renko Geffarth und Markus Meumann, Berlin/Boston 2013, S. 322-339, hier S. 329; zur Forschung S. 326-328.

11 So Ursula Geitner: Klartext. Zur Physiognomik Johann Caspar Lavaters, in: Geschichten der Physiognomik. Text - Bild - Wissen, hg. von Rüdiger Campe und Manfred Schneider, Freiburg i.Br. 1996, S. 357-385, hier S. 358. 
Faszination für ein transparentes Verhältnis zwischen innen und außen, wenn er den Gedanken entfaltet, "aus Teilen und Zügen des Gesichts Kräfte und Bewegungen eines Geistes«, "aus dem Laut den Gedanken [entbinden] « zu können $(\mathrm{I} / 5,182)$. Andererseits sind Körper und Geist für ihn inkommensurable Größen. ${ }^{12}$

Die physiognomische Grundannahme, dass von den Zeichen auf die Bedeutung geschlossen werden könne, wird im Komet bereits mit dem Namen des Helden, dem "Namen des Buchs" $(\mathrm{I} / 6,568)$, unterminiert: Wo Marggraf draufsteht, ist nicht (mit Sicherheit) ein Markgraf drin. ${ }^{13}$ Nicht von ungefähr alludiert Jean Paul den locus ab etymologia, den Topos, "vom Eigennamen Rückschlüsse" auf den Charakter zu ziehen. ${ }^{14}$ In diesem Sinn berührt sich eine mit dem Namen aufgerufene "Mimologik « mit der physiognomischen Entzifferung. ${ }^{15}$

Der Name des Helden ist als Homophonie von Eigen- und Gattungsname doppelsinnig. Mit ihr werden im Komet die Referenz und damit einhergehend die Identität des Helden problematisch. Der »Kling-Witz« (I/5, 191) führt wie zahlreiche andere "Namen-Ähnlichkeit[en] $(\mathrm{I} / 6,600)$ zu komischen Missverständnissen: So betont der Erzähler, dass dem Helden die »uneigentlichen Namen" unzugänglich sind: "Nikolaus [dachte] nie bei seinem Geschlechtnamen an den regierenden Markgraf«, weshalb er, wenn er vom Markgrafen reden hört, glaubt, "man habe bloß ihn selber gemeint anstatt des Markgrafen« (I/6, 632).

Kennt der Held nur Eigennamen, sind diese im anagrammatischen Spiel Jean Pauls die eigentlichen Namen. Sofern Nikolaus aber gerade die uneigentliche Dimension kassiert, ist seine idiosynkratische Sprache der Eigennamen Modellen einer ursprünglich metaphorischen Sprache, wie sie das 18. Jahrhundert und Jean Paul konzeptualisieren (vgl. I/5, 184), diametral entgegengesetzt. Nikolaus hat keinen Zugang zur Etymologie, die entweder im etymon die wahre Bedeutung vermutet oder Einsicht in das unendliche Verweisungssys-

$12 \mathrm{Zu}$ den widersprüchlichen Auffassungen siehe Kurt Wölfel: »Ein Echo, das sich selber in das Unendliche nachhallt«. Eine Betrachtung von Jean Pauls Poetik und Poesie, in: ders.: Jean Paul-Studien, hg. von Bernhard Buschendorf, Frankfurt a.M. 1989, S. 259-300, bes. S. 272f.

13 Jean Paul spielt mit der intrikaten Frage, ob Namen auch Bedeutung zukommt. Siehe hierzu Stefan Sonderegger: Die Bedeutsamkeit der Namen, in: Zeitschrift für Literaturwissenschaft und Linguistik 67 (1987), S. 11-23, bes. S. 15 und 18.

14 Wolfram Groddeck: Reden über Rhetorik. Zu einer Stilistik des Lesens, 2. durchges. Aufl., Frankfurt a.M. 2008, S. 53.

15 Gérard Genette: Mimologiken. Reise nach Kratylien, übers. von Michael von KillischHorn, Frankfurt a.M. 2001, S. 10. 
tem zwischen den Zeichen gewährt. ${ }^{16}$ Während Nikolaus im Reich der Zeichen ohne Orientierung umherirrt, schafft Jean Paul ein textuelles Universum von Ähnlichkeiten. ${ }^{17}$ So bildet die minimale Verschiebung der Grapheme $\langle\mathrm{g}\rangle$ und $\langle\mathrm{k}\rangle$ die Handlung vor und reflektiert die Semiosis im Komet auf der Mikroebene. ${ }^{18}$

Wie der Held von der etymologischen Tiefendimension der Sprache abgeschnitten ist, ist er auch ein entwurzeltes Subjekt. Nikolaus, der den "Namen seines Herrn Vaters" $(I / 6,889)$ nicht kennt, ist allein auf die Untiefen seiner Phantasie verwiesen: Sein Fenster zur Wirklichkeit ist ob seiner Annahme einer vermeintlich eindeutig referierenden Sprache imaginär verhangen. Ist Nikolaus so ein Erbe von Cervantes' »Don Quixote« (I/6, 569), besteht seine Narrheit darin, dass er die Unterscheidung zwischen Schein und Wirklichkeit zugunsten der fixen Ideen suspendiert, der Nachfolger des heiligen Nikolaus oder ein Fürst zu sein. ${ }^{19}$ Blumenberg hat angemerkt, dass im Komet die »Darstellung" der Vertauschbarkeit von »Illusionäre[m] « und »Reale[m] « Thema ist, wobei die »Doppelpoligkeit von [...] Gegenstand und Zeichen" zerbrochen werde. ${ }^{20}$ Damit wird auch Jean Pauls zentrale Frage, ob das »Ich eine [...] lesbare Wirklichkeit vor[findet] $\ll^{21}$ virulent: Wie wird unter den Vorzeichen einer Welt der Fassaden mit `Nichts dahinter überhaupt physiognomiert? Der Komet variiert die Bestimmung der Physiognomik aus dem Magie-Aufsatz. ${ }^{22}$ Wird dort die sichtbare Welt durch »Physiognomik« beseelt, indem die eigene

16 Vgl. Stefan Willer: Orte, Örter, Wörter. Zum locus ab etymologia zwischen Cicero und Derrida, in: Rhetorik. Figuration und Performanz, hg. von Jürgen Fohrmann, Stuttgart/ Weimar 2004, S. 39-58, bes. S. 42.

17 So macht Jean Paul den `Zusammenhang der Dinge durch Aufzeigen von Ähnlichkeiten entsprechend dem Witz als "spielende[m] Anagramm der Natur" $(\mathrm{I} / 5,47)$ sichtbar, wo er anlässlich seiner komischen Geschichte im Zeichen der »epische[n] Flügelschnecke« den »Name[n] der geschichtlichen Muse« Klio aus demjenigen des naturhistorischen Wurms »Clio« in seiner poetischen Etymologie ableitet $(\mathrm{I} / 6,656)$.

18 Vgl. Götz Müller: Mehrfache Kodierung bei Jean Paul, S. 88; zum Verhältnis von Identität und Differenz in der klassischen Episteme sowie zum Namen vgl. Oliver Simons: Jean Pauls imaginäre Epistemologie, S. 181 und 184.

19 Vgl. hierzu Maximilian Bergengruen: Pol und Gegenpol eines Magneten. Zwei Studien zu Jean Pauls Konzept der Doppelautorschaft in Siebenkäs, Flegeljahren und Komet, in:Jahrbuch der Jean-Paul-Gesellschaft 45 (2010), S. 45-79, bes. S. 52-69.

20 Hans Blumenberg: Wirklichkeitsbegriff und Möglichkeit des Romans, in: ders.: Ästhetische und metaphorologische Schriften. Auswahl und Nachwort von Anselm Haverkamp, 4. Aufl., Frankfurt a.M. 2014, S. 47-73, hier S. $65 f$.

21 Monika Schmitz-Emans: Der verlorene Urtext. Fibels Leben und die schriftmetaphorische Tradition, in: Jahrbuch der Jean-Paul-Gesellschaft 26/27 (1992), S. 197-222, hier S. 200. Bereits Schmitz-Emans charakterisiert den "späten Roman als erzählerische Paraphrase des Magie-Aufsatzes«. Monika Schmitz-Emans: Der Komet als ästhetische Programmschrift. Poe- 
Seele »in fremde Augen, Nasen, Lippen« projiziert wird (I/4, 203f.), so nimmt der homo ludens Nikolaus im Komet X für Y (vgl. I/6, 963)23 oder setzt »wie ein Schauspieler« zu den Bedingungen seiner projizierenden "Phantasiekraft« die "fremde [Seele] an die Stelle der seinigen" (I/6, 590). Lavaters Physiognomik dient dabei insofern zur Reflexion der Phantasie, als Nikolaus' mimetische Fähigkeit gerade dadurch illustriert wird, dass er sich "zwei Sonntage hintereinander für Kaspar Lavater den zweiten« hält (I/6, 590f.).

\section{Körper: «bloß der Nase nach»}

Gesichter und partiale Körperzeichen sind im Komet Schauplatz physiognomischer (De-)Chiffrierung. Insbesondere der großen Nase des Helden kommt im Komet eine Leitfunktion zu. Die Nase ist ein von Lavater nobilitiertes Körperzeichen (vgl. F IV, 257), an dem sich auch die Physiognomik-Satire von Musäus, Lichtenberg, aber auch schon Sterne abarbeitet. Sie rückt bereits im »Ur- oder Belehnkapitel« $(\mathrm{I} / 6,574)$ ins Zentrum, in dem die Vorgeschichte von Nikolaus' Eltern erzählt wird. Henoch Elias Marggraf, ein geiziger Apotheker, lernt in Margarethahausen die Sängerin Mara kennen, die er prompt heiratet. Mara begeht jedoch noch im Kurort einen Seitensprung mit einem Fürsten von beachtlicher Nase mit Pockennarben. Der Held Nikolaus Marggraf hat eben eine solche pockennarbige Nase, die drei physiognomische Interpretationen erfährt: Im Erzählerdiskurs wird sie als generisches Merkmal, in der erzählten Welt als religiöses und ökonomisches Versprechen gedeutet.

Dass die Nase des kleinen Nikolaus groß ist, bezeichnet qua Ähnlichkeit nicht nur seinen vermutlichen fürstlichen Vater, sondern vermeintlich auch dessen Herkunft. Hierfür konnte sich Jean Paul auf Lavaters Fragment Ein Wort über die Nase beziehen, das den »Italiaener[n]«»[g]roße und bedeutende Nasen" attestiert, ebenso wie den "Franzosen", die seines Erachtens "den Charakter ihrer Groeße am meisten in den Nasen« haben (F IV, 258). Mit seiner "großen welschen Nase« $(\mathrm{I} / 6,585)$ wäre Nikolaus gemäß der von Jean Paul in der Vorschule vorgenommenen Unterscheidung von drei Romanschulen nicht etwa als bürgerlicher Held der "deutschen " oder "niederländischen" $(\mathrm{I} / 5,254)$, sondern als adeliger »der italienischen Schule» ausgewiesen, deren

tologische Konzepte, Aporien und ein Sündenbock, in: Jahrbuch der Jean-Paul-Gesellschaft 35/36 (2000/01), S. 59-92, hier S. 68.

Vgl. Max Kommerell: Jean Paul, 5., durchges. Aufl., Frankfurt a.M. 1977, S. 372. 
Programm »Erhabnes" vorsieht $(\mathrm{I} / 5,253)$. In der Tat erhebt die Nase ihn satirisch zu allem Großen und Erhabenen in der Einbildung (vgl. I/6, 589); ist doch die Nase in Lavaters `Nasen-Fragment die "Wiederlage des Gehirns", weil »auf ihr [...] alle die Kraft des Stirngewoelbes zu ruhen« scheint (F IV, 257). Daneben erklärt Jean Paul die vermeintliche Erhabenheit seines Helden mit der Phrenologie Galls, die anhand des äußeren Schädels auf Fähigkeiten des Gehirns schließt und von einer Entsprechung zwischen »bestimmte[n] Erhabenheiten" und »bestimmten Eigenschaften" ausgeht: ${ }^{24}$ "[D]ie beiden Gehirnhügel« müssen sich »nach Gall, wie zwei Parnaßspitzen innen [...] folglich außen sehr erhoben haben" $(\mathrm{I} / 6,602)$. In einem Kommentar zum Genre seines Buches greift Jean Paul indes auf Carl Friedrich Benkowitz' Studie Der Messias von Klopstok [sic] zurück. Obgleich Benkowitz "richtig« bemerke, »daß ein Heldengedicht wie die Messiade die Nase als ein zu gemeines Wort [...] auslasse«, ${ }^{25}$ ist es für Jean Paul gerade »dieses alltägliche Gliedmaß«, welches »des Reiseapothekers gemeines Leben zum Epos, zum Pik mit Nasenlöchern" erhebt (I/6, 583). Die Nase - Schneise zwischen "Geist und Sinnlichkeit« ${ }^{26}$ - ist als großes "vorgewölbte[s] Körperteil[] «, erst recht aber mit den zwei kratergroßen »Körperhöhlen« freilich auch das groteske Körperzeichen schlechthin. ${ }^{27}$ Die erhaben-groteske Nase des Helden ist demnach im Kalkül Jean Pauls ein 'generisches Körperzeichen. Ist der Komet eine Parodie des Fürstenromans, insofern sich ein sdeutscher Held und Erbe der sniederländischen< Idylliker im Pattern des hohen Romans wiederfindet, ${ }^{28}$ so greift Jean Paul gerade zur (Doppel-)Physiognomie des Helden, zum "seltsame[n] Ineinanderbau von welschem und deutschem Gesicht« (I/6, 589), zur signifikant doppelsinnigen Nase, um dieses generische Arrangement ins Bild zu bannen.

24 Franz Joseph Gall: Des Herrn Dr. F. J. Gall Schreiben über seinen bereits geendigten Prodromus über die Verrichtungen des Gehirns der Menschen und der Thiere, an Herrn Jos. Fr. von Retzer, in: Der Neue Teutsche Merkur, December 1798, S. 311-332, hier S. 324f.

25 Vgl. Carl Friedrich Benkowitz: Der Messias von Klopstok, aesthetisch beurtheilt und verglichen mit der Iliade, der Aeneide und dem verlohrnen Paradiese. Eine Preisschrift, die von der Amsterdamer Gesellschaft zur Beförderung der schönen Künste und Wissenschaften eine doppelte Medaille erhalten hat, Breslau 1797, S. 146.

26 Albrecht Koschorke: Zur Kulturgeschichte der Nase. Entsprechungen zwischen Gesichtsund Körperordnungen, in: Gesichter. Kulturgeschichtliche Szenen aus der Arbeit am Bildnis des Menschen, hg. von Sigrid Weigel, München 2013, S. 187-198, hier S. 195.

27 Michail Bachtin: Rabelais und seine Welt. Volkskultur als Gegenkultur, übers. von Gabriele Leupold, hg. von Renate Lachmann, Frankfurt a.M. 1987, S. 381, vgl. S. 357-359.

28 Siehe zur Konfrontation von bürgerlichem und fürstlichem Romanmuster auch Maximilian Bergengruen: Pol und Gegenpol eines Magneten, S. 51. 
Die Nase selbst ist im Komet ihrerseits Trägermedium von Körperzeichen, die weitere physiognomische Lektüren in Gang setzen. Denn Nikolaus hat die "medizinische Merkwürdigkeit[] ] von "zwölf Blatternarben", die er "auf die Welt gebracht, als hätt' ihn die Natur schon ungeboren mit diesen Stigmen (Wundenmalen) [...] tätowiert« (I/6, 577f.).

Mit den angeborenen Malen alludiert Jean Paul Paracelsus' Gedankenfigur der impressiones, ${ }^{29}$ auf die sich auch Lavaters Deutung der "Muttermaehler" als Effekt der »Einbildung der Mutter [...] auf ihre Leibesfrucht« bezieht (F IV, 66). Als impressiones ‘ werden Nikolaus' Blatternarben in der Tat vorstellig, wenn Mara sie auf den visuellen Eindruck, den der Fürst auf sie gemacht hat, zurückführt (vgl. I/6, 578). Dabei verschränkt der Erzähler den ’hermetischen< Diskurs mit demjenigen der Heredität: Als ein Mönch Mara die letzte Beichte abnimmt, hört Henoch, »daß ihr Nikolaus der Sohn eines katholischen weltlichen Fürsten sei, [...] der eben seinen Heiligenschein und seine Nasen-Narben auf den Kleinen fortgepflanzt« habe (I/6, 580). In der Deutung Maras sind die Narben als Stigmata gemäß der christlichen Ikonografie Zeichen der Heiligkeit, die Nikolaus durch die Zwölfzahl als 'Postfiguration des Messias kennzeichnen. Als Male wie als Stigmata sowie durch die explizite Metaphorik des Tätowierens werden die Narben dabei zu Körperzeichen zwischen Schrift und Bild, zu einer in den Körper eingetragenen Bilder- bzw. Hieroglyphenschrift. Ikonizität kommt ihnen auch insofern zu, als Mara sie als »Titelvignetten« bzw. "Buchdruckerstöcke« des Heiligenlebens von Nikolaus liest (I/6, 578). Mit den Narben als »durch Abklatschen [...] vervielfältigtes Bild«, als Randverzierung, die man "auf gedruckten Bücherumschlägen, auf Titeln « findet, ${ }^{30}$ wird Nikolaus buchstäblich zum büchernen Helden, der dem Programm seiner paratextuellen 'Narben-Vignetten in seinem Heiligspielen auch nachkommt. Wo aber Physiognomik und Heredität in der Metaphorik der Druck-Reproduktionstechnik enggeführt werden, erweist sich die Physiognomie gerade nicht mehr als individuelle. Zumindest garantieren die Narben keine stabile Identität im Zeichen des unterscheidenden Merkmals: Mögen sie auch im Sinn der von Lavater erörterten "Aehnlichkeit der Aeltern und Kinder" (F IV, 326-340) eine Ähnlichkeit zum vermeintlichen Vater aufzeigen, dieser bleibt trotz

29 Vgl. Paracelsus: De Natura Rerum, in: ders.: Bücher und Schriften, hg. von Johannes Huser [Basel 1590], Faksimileausgabe mit einem Vorwort von Kurt Goldammer, Hildesheim 1971-1975, Bd. 6, S. 255-362, hier S. 332f.

30 Art. "Buchdruckerstock, Stock, Vignette«, in: Encyklopädie der Buchdruckerkunst, bearbeitet von Hermann Neubürger, Leipzig 1844, S. 51; vgl. Art. "Vignette«, in: Jacob und Wilhelm Grimm: Deutsches Wörterbuch, Bd. 26, Leipzig 1951, Sp. 357. 
identifizierender Merkmale dunkel. Deutet Mara die Narben im Register der Esoterik und Religiosität, so spielt sich dabei semiotisch gelesen auf der Nase zugleich eine ganze Kulturgeschichte des Zeichens ab. ${ }^{31}$ Indem die Narben im Erzählerkalkül als Male, impressiones, als Vignette, Tätowierung und Stigmata, als indexikalische und ikonische Zeichen zugleich apostrophiert werden, geraten die textuellen Narben nicht nur zum Kreuzungspunkt von Diskursen, sondern auch zum Reflexionsmedium von Signifikation. ${ }^{32}$

Die Pointe Jean Pauls ist indes das Spiel mit den Narben als den indexikalischen Zeichen, für die der Zusammenhang von Ursache und Wirkung gilt, demzufolge sie auf eine vorgängige Wunde verweisen. Auf diese Relation weist Jean Paul mit der Bemerkung hin, dass Nikolaus die Blatternarben "auf die Welt gebracht, als hätt' ihn die Natur schon ungeboren" markiert, "was aber nicht gewesen sein kann, da er später die wahren Pocken bekam und also die Narben früher als die Wunden hatte« $(\mathrm{I} / 6,578)$. Im figurierten hysteron proteron sind Nikolaus durch den rallmächtigen Vater Jean Paul (vgl. $\mathrm{I} / 6,584)$, der den Komet "geschaffen und gezeugt « hat $(\mathrm{I} / 6,567)$, so schlechtweg unmögliche Narben in seinen fiktiven Leib eingeschrieben: Nikolaus ist mit seinen Körperzeichen durch und durch Zeichenkörper. ${ }^{33}$ Dem entspricht in Jean Pauls programmatischem Kommentar im 'Ur- oder Belehnkapitek die Inversion des Begründungszusammenhangs von Natur und Fiktion: "natura fictionem sequatur« $(\mathrm{I} / 6,584)$. Die Narben markieren Literatur so als selbstreferentielles Medium, das die eigene Fiktionalität ausstellt.

Diese textinterne 'Fiktionalisierung` der Körperzeichen geht nicht zuletzt mit der Semiotik des Geldes einher, auf die in der Deutung des homo oeconomicus Henoch referiert wird. Mit der Narben-Nase steht neben der Vererbung geistiger und physischer Merkmale auch die Aussicht auf eine Erbschaft zur Debatte. Hatte Henoch bereits Verdacht geschöpft, von Mara gehörnt worden zu sein, als er die Physiognomie seines langnasigen Sohnes mit seiner eigenen und derjenigen seiner beiden kurznasigen Töchter vergleicht, so bestätigt ihn

31 Zur Semiotik der Narbe vgl. Dagmar Burkhart: Narbe. Archäologie eines literarischen Motivs, in: Arcadia 40/1 (2005), S. 30-60, bes. S. 33-38.

32 Mit den Zeichen auf der Haut konstelliert Jean Paul überdies die zentralen Figuren Nikolaus, Worble und Kain: Nikolaus' Pendant Worble hat, wie andere Humoristen, Blatternarben, die allerdings »kein Zeichen" sind (I/6, 646), sein Gegenspieler Kain trägt indes das Kainszeichen.

33 Damit steht er in der Nachfolge Christi und dessen »Körper als Buchseite«, gemäß Urban Küsters: Zeichen auf der Haut in der religiösen Kultur des Mittelalters, in: Literalität und Körperlichkeit. Littéralité et Corporalité, hg. von Günter Krause, Tübingen 1997, S. 47-53, hier S. 47. 
Maras Beichte, die er gelassen nimmt. Denn ihm winkt nicht allein das Erbe fürstlicher »Juwelen« (I/6,580), er hofft zudem auf Entschädigung durch den Fürstvater. »Über das künftige Auftreiben eines Vaters« ist er "gar nicht in Angst«: "Ich gehe‘, dacht' er, ’bloß der Nase nach, nämlich der fürstlichen pockennarbigen « $(\mathrm{I} / 6,582)$. Er investiert in Nikolaus als "Ziehbrunnenkette in die Zukunft« $(\mathrm{I} / 6,582)$, aus dessen Physiognomie er Kapital zu schlagen hofft. Henoch organisiert eine billige und inadäquate Fürsten-Erziehung (vgl. I/6, 616 und 618), denn er will den Fürstsohn nicht nur »so früh als möglich [...] auf den Kauf gemacht haben", sondern »auch künftig recht viel für das Erziehen einnehmen« $(\mathrm{I} / 6,618)$. Was die Physiognomie für Lavater im übertragenen Sinn ist, ist sie für Henoch ganz buchstäblich: ein "Capitalfond« (F IV, 39). Deshalb führt er ein »Kapitalbuch« (I/6, 642) und lässt entsprechend der Zinslogik die Zeit für sich arbeiten (vgl. I/6, 582 und 621). Wenn Nikolaus mit seiner Narben-Prägung zur "[P]fandschaft« (I/6, 621) wird, dann rekurriert Jean Paul u.a. auf das Pfand in der Aufklärungsökonomie. Insofern etwa Law das Papiergeld als »Bodengeld« bestimmte, ging mit ihm eine "Sicherheitsgarantie« einher. ${ }^{34}$ Das "Zeichen-Geld" des 18. Jahrhunderts hatte demnach insofern "Pfandcharakter", als es durch eine Wertsubstanz gedeckt war und die materiale Einlösung versprach. Die Zeit nach 1797 markiert dabei die Phase, an dem das Zeichen-Geld allmählich in »Kreditgeld« überführt wird, mit dessen Selbstreferentialität der Nimbus des nur "Scheinhaften« einhergeht. ${ }^{35}$ Nikolaus' Physiognomie kommt demnach auf Henoch wie Papiergeld, von dem dieser nicht weiß, ob es durch Substanz gedeckt ist: als "fiktives Zeichen ohne gesicherte Bedeutung « ${ }^{36} \mathrm{Als}$ Methode, mit der Lavater aus dem menschlichen Antlitz auf die göttliche Offenbarung schließt, hat Physiognomik für Henoch ausgedient. Sie erfährt eine Umdeutung: Das Erkenntnisinteresse richtet sich nicht mehr auf das innere Wesen, sondern im Rahmen des konjekturalen ökonomischen Wissens auf den Geldwert: Geld tritt an die Stelle des (Heiligen) Geistes. ${ }^{37}$

34 John Law: Betrachtungen über das Geld und den Handel einschließlich eines Vorschlags zur Geldbeschaffung für die Nation $(1705,1720)$, in: ders.: Handel, Geld und Banken, übers. und mit einem Nachwort hg. von Achim Toepel, Berlin 1992, S. 11-125, hier S. 96 und 85 .

35 Joseph Vogl: Kalkül und Leidenschaft. Poetik des ökonomischen Menschen, 4. Aufl., Zürich 2011, S. 318 und 323.

36 Jacques Derrida: Falschgeld. Zeit geben I, übers. von Andreas Knop und Michael Wetzel, München 1993, S. 124.

37 Zur »Geschichte der monetären Ablösung religiöser Integrationsformeln« vgl. Jochen Hörisch: Kopf oder Zahl. Die Poesie des Geldes, Frankfurt a.M. 1996, S. 32. Jean Paul inszeniert so mit der Physiognomik, wie auch die Leichenpredigt auf die Magd Regina 
Es wird deutlich, dass Jean Paul Physiognomik, Religion und Ökonomie so verschaltet, ${ }^{38}$ dass sie je nur unsicheres Wissen produzieren: Wie die Physiognomie und der religiöse Text muss auch das "ökonomische Zeichenspiel « interpretiert werden. ${ }^{39}$ Wo aber spekuliert wird, geht es um unsichere Prognosen. Während sich Henoch ob des physiognomischen Pfands »seines möglichen Lohns [...] gewiß (I/6, 623) wähnt und es auch Nikolaus »das gewisseste « ist, dass er seinen "Vater mit der Pockennarbennase [...] finden werde« (I/6, 652), antwortet der Roman auf diese Gewissheit bekanntlich damit, dass er abbricht, noch bevor es zum Finden kommt. In diesen Abbruch wäre auch eine Antwort auf das Begehren der Physiognomik nach der Sistierung »transcendente $[\mathrm{r}]$ Bedeutung « ${ }^{40}$ hineinzulesen. In dem Maße, wie die physiognomische Suche auch eine nach letztgültigen Referenzen ist, wäre sie unabschließbar, breche man anders nicht ab (vgl. I/6, 657, Anm. 1). Indes: Wo Henoch aus der Masken-Physiognomie des Humoristen Worble falsche Schlüsse zieht (vgl. I/6, 646), lässt Jean Paul schon textintern keinen Zweifel daran, dass Physiognomik ein Quell der Irrtümer ist.

\section{Gouvernementale Schrift: ıMenschentitelblatt Passı}

Nach alchemistischen Versuchen, bei denen ein künstlicher Diamant gelingt, durch den er zu Reichtum gelangt, zieht Nikolaus als Parodie des 'verborgenen Prinzen ${ }^{41}$ mit einem Hofstaat aus, um seinen Fürstenvater und die Prinzessin Amanda zu suchen. Nikolaus wählt als Reise-Pseudonym den "grotesken Inkognito-Namen" (I/6, 894) Graf "Hacencoppen " (I/6, 896), und der Reisemarschall Worble kauft auf dem »Polizeiamt« kurzerhand einen gefälschten Pass (I/6, 898).

erhellt, sowohl die zunehmende Verdinglichung als auch deren Rache durch metaphorische Verdinglichung (vgl. I/6, 1022-1032).

38 Zum Bildfeld der Ökonomie bei Jean Paul vgl. auch Caroline Pross: Falschnamenmünzer. Zur Figuration von Autorschaft und Textualität im Bildfeld der Ökonomie bei Jean Paul, Frankfurt a.M. 1997.

39 Joseph Vogl: Das Gespenst des Kapitals, 6. Aufl., Zürich 2012, S. 23.

40 Georg Christoph Lichtenberg: Ueber Physiognomik; wider die Physiognomen. Zu Befoerderung der Menschenliebe und Menschenkenntniß. Zweyte vermehrte Aufl., Goettingen 1778, S. 81.

41 Götz Müller: Der verborgene Prinz. Variationen einer Fabel zwischen 1768 und 1820, in: Jahrbuch der Jean-Paul-Gesellschaft 17 (1982), S. 71-89, bes. S. 88f. 
Jean Paul nimmt dies zum Anlass für eine Digression über die Notwendigkeit des »Menschentitelblatt[s], Paß genannt« (I/6, 898). Das »kurze[] schwache Lob der jetzigen höhern Paßwissenschaft « (I/6, 900) referiert auf die Veränderungen des Passwesens und die Einführung von Kontrollpraktiken nach 1792 und in der nachnapoleonischen Restauration. ${ }^{42}$ Jean Paul widmet sich satirisch der Frage, ob der »vollständig bestimmende[] Zettel " gegen »die menschliche Würde" sei $(\mathrm{I} / 6,900)$, weil er als »vorausdatierte[r] Steckbrief [ ] $(\mathrm{I} / 6,901)$ den Menschen als Verbrecher stigmatisiere. Er argumentiert im Gegenteil, dass der Pass "außer Landes" die »Ehrlichkeit" des "Passagier[s] « allererst bezeuge $(\mathrm{I} / 6,898)$. So könne man, da man an »jeder Grenze« unter dem Verdacht stehe, »ein mutmaßlicher Spitzbube« zu sein, jetzt den »Paß als Ablaßbrief « hervorziehen und "das göttliche Ebenbild« wieder erneuern $(\mathrm{I} / 6,898)$. Im Zuge dieser Satire der gouvernementalen Sicherheitsmaßnahmen (vgl. I/6, 902) ${ }^{43}$ beschreibt der inszenierte Autor auch sein eigenes Pass-»Signalement« $(\mathrm{I} / 6,900)$ : »[I]ch zeige meinen gestempelten Papier-Paß vor, worin (außer meiner Handschrift) steht, daß ich 5 Fuß und 10 Zoll lang bin, 59 Jahre alt, in Wunsiedel geboren etc., daß meine Stirn breit und hoch ist und mein Mund klein" (I/6, 900f.). Das Signalement des textinternen Jean Pauk verweist auf den extratextuellen Johann Paul Friedrich Richter, der bei Erscheinen des dritten Bandes seines Spätwerks 59 Jahre alt ist. Bei den Daten der Gesichtsbeschreibung handelt es sich freilich um das Zitat der Physiognomie eines sgroßen Kopfes<: Mit der vernachlässigten unteren, sinnlich-animalischen Gesichtshälfte und dem Topos der hohen und breiten Stirn ${ }^{44}$ schreibt sich Jean Paul in seinen Text als Autor von großen Geisteskräften ein.

Anhand des Signalements fragt Jean Paul nach der Rolle, die die Physiognomik für die Repräsentation spielt. Denn die Passszene ist die Szene der Repräsentation, insofern der »Paßinhaber« $(\mathrm{I} / 6,901)$ »an der Grenze beim

42 Vgl. John Torpey: The Invention of the Passport. Surveillance, Citizenship, and the State, Cambridge University Press 2000, S. 21-56 und 57-66, bes. S. 63; Hannelore Burger: Das Paßwesen, in: Grenze und Staat. Paßwesen, Staatsbürgerschaft, Heimatrecht und Fremdengesetzgebung in der österreichischen Monarchie 1750-1867, hg. von Waltraud Heindl und Edith Saurer, Wien/Köln/Weimar 2000, S. 3-87.

$43 \mathrm{Zu}$ den "Sicherheitsmechanismen« und der "Polizei« als Merkmal moderner Gouvernementalität vgl. Michel Foucault: Sicherheit, Territorium, Bevölkerung. Geschichte der Gouvernementalität I, Vorlesung am Collège de France 1977-1978, übers. von Claudia Brede-Konersmann und Jürgen Schröder, 4. Aufl., Frankfurt a.M. 2015, S. 449-519, hier S. 506 und 462

44 Vgl. Albrecht Koschorke: Zur Kulturgeschichte der Nase, S. 190. 
Vorzeigen [...] seines Reisepasses" nicht nur sich selbst, sondern auch seine durch den Pass »legitimierte " und autorisierte Präsenz präsentiert. ${ }^{45}$ Dies spielt Jean Paul narratologisch aus, denn mit der Autofiktion des Passes markiert er die ontologische Grenze, die in jeder Erzählung zwischen dem realen Autor und dessen textinterner Repräsentanz eingezogen ist. Sofern diese das 'Bild des Autors ist, ${ }^{46}$ "wie es sich [...] auf der Basis des Textes konstruieren" lässt, ${ }^{47}$ bringt Jean Paul die strukturellen Voraussetzungen des Erzählens wie der Rezeption an der Textoberfläche zum Vorschein.

Die Daten eines Passes hätten freilich eindeutig auf das Referenzobjekt zu passen, damit der Pass seinen Zweck erfüllt, nämlich personale Identität festzustellen. Dabei spielt Jean Paul mit den Schwierigkeiten, die sich aus einer Personenbeschreibung ergeben, die immer uneindeutig bleiben muss. ${ }^{48}$ Dass es "einen Spitzbuben geben könnte, auf welchen alles von mir so passete, daß wir einander deckten«, stellt Jean Paul in Abrede: »Unmöglich! - Sogar meine nächsten Nachahmer und Diebe würde mein Paß, so sehr ich auch Swift und Sterne nachgeahmt und bestohlen, auf der Stelle unterscheiden von mir« (I/6, 901). Während der Erzähler über die eindeutige Zuordenbarkeit der Data im Pass zum Referenzobjekt reflektiert, hat er dem Leser in der intertextuellen Textpraxis längst eine lange Nase gezeigt. Denn Jean Paul hat zugleich Sterne's Sentimental fourney und Swift's A Modest Proposel splagiiert ${ }^{49}$ Durch Ambiguierung der Zeichen und Referenzen wird die behauptete Eindeutigkeit seines literarischen Passes unterminiert. Während seine Figur affirmativ die Unterscheidung zwischen Zeichen und Bezeichnetem tilgt, behauptet Jean Pauk eine unhintergehbare Differenz eben auch da, wo splagiierend wiederholt wird.

Während Jean Paul im Pass-Digress über Signifikation, Referenz und eine paradox begründete Originalität reflektiert, setzt er zugleich eine zeitkritische

45 Louis Marin: Das Sein des Bildes und seine Wirksamkeit, in: Das Bild ist der König. Repräsentation nach Louis Marin, hg. von Vera Beyer, Jutta Voorhoeve und Anselm Haverkamp, München 2006, S. 15-23, hier S. 17.

46 Vgl. Wayne C. Booth: The Rhetoric of Fiction, 2. Aufl., Chicago/London 1983, S. 75.

47 So Booths Kritiker Gérard Genette: Die Erzählung, übers. von Andreas Knop, 2. Aufl., München 1998, S. 285. Zur Gesichtsbeschreibung als Eintragungsort des Autors vgl. auch (mit anderen Argumenten) Peter von Matt: ... fertig ist das Angesicht. Zur Literaturgeschichte des menschlichen Gesichts, Frankfurt a.M. 1989, S. 115.

48 So Johann Gottlieb Fichte: Sämtliche Werke, hg. von Immanuel Hermann Fichte, Bd. 3, II. Abt. Bd. 1, A: Zur Rechts- und Sittenlehre, Berlin 1845, S. 295.

$49 \mathrm{Zu}$ Sterne vgl. Stephanie DeGooyer: The Poetics of the Passport in A Sentimental fourney, in: Sterne, Tristram, Yorick: Tercentenary Essays on Laurence Sterne, hg. von Melvyn New, Peter de Voogd und Judith Hawley, University of Delaware Press 2015, S. 201-218. 
Pointe. Der Möglichkeit des gefälschten Passes, der Tücke des scheinhaften Zeichens könne die Polizei dadurch begegnen, dass sie dem Menschen den Pass wie einem Vieh das Brandzeichen in die Haut »[t]ätauiere[ ]« (I/6, 902), um so durch »Brandmarken« (I/6, 903) die Kluft zwischen Zeichen und Bezeichnetem zu überbrücken. ${ }^{50}$ Dergestalt überbietet Jean Paul die polizeiliche 'Prosopographie durch Dermatographie, bei der die Zeichen auf dem Körper als signifikante »Kehrseite« sicher den »Gehalt [ ] bezeichnen (I/6, 903). Literarische Physiognomik reflektiert so die Konsequenzen des verwaltenden Zugriffs des modernen Staates auf das Subjekt. Mit der satirischen Kritik an der Inhumanität der zunehmenden Bürokratisierung, die das Subjekt vermisst, verzeichnet und sistiert, dekuvriert Jean Paul zugleich die Implikationen einer Physiognomik im Zeichen despotischer Vernunft, indem er ihr Begehr nach einem gläsernen Menschen radikal weiterdenkt.

\section{Bilder: Puppenliebe und Hacencoppens physiognomische Fragmente}

Die Reflexion der physiognomischen Körper- und Schriftzeichen steht überdies im "Spannungsfeld divergenter Bild-Konzepte und Bild-Diskurse, 51 die im dritten Band des Komet zunehmend virulent werden. Jean Paul macht sich dabei die Polyvalenz des Bild-Begriffs ${ }^{52}$ zur Selbstbeobachtung seines Schreibens zunutze. Hierfür konnte er auf die Physiognomik Lavaters insofern zurückgreifen, als dessen Thema "zwar die menschliche Physiognomie« war, "aber das Material, anhand dessen er seine physiognomische Wissenschaft entwickelte, $[\ldots]$ hauptsächlich aus der Kunst« stammte. ${ }^{53}$

In der Kindheitsgeschichte des Helden begegnen Puppen, auf die Nikolaus das Theologumenon der Gottebenbildlichkeit überträgt. Damit traktiert er sie in dem Maße physiognomisch, wie Lavater die Physiognomik theologisch

50 Zu den Swift-Bezügen vgl. auch Daniel Bowles: The Ends of Satire. Legacies of Satire in Postwar German Writing, Berlin/München/Boston 2015, S. 38f.

51 Monika Schmitz-Emans: Bildlichkeit, Bilder und bildende Kunst bei Jean Paul, in: Jean Paul, der Fremde. Kleine Vorschule zu Texten und Kontexten eines schwierigen Autors, hg. von Gunnar Och und Georg Seiderer, Würzburg 2014, S. 83-102, hier S. 99.

52 Was Bild sein kann, wird im Komet aufgeboten: grafische, optische, perzeptuelle, innere und literarische Bilder; vgl. W.J.T. Mitchell: Was ist ein Bild, in: ders.: Bildtheorie, hg. und mit einem Nachwort von Gustav Frank, Frankfurt a.M. 2008, S. 15-77, hier S. 20. Daniela Bohde: Physiognomische Denkfiguren in Kunstgeschichte und visuellen Wissenschaften, S. 93. 
fundiert: "Gott schuf den Menschen sich zum Bilde!« (F I). ${ }^{54}$ Der Titel des ersten Kindheitskapitels erinnert zudem an Lavaters Beförderung der [...] Menschenliebe: "wie der kleine Nikolaus die Menschen sehr zu lieben weiß « $(\mathrm{I} / 6,585)$. In der Tat hält Nikolaus sich mit seiner Fähigkeit zur mimetischen Verähnlichung auch für Lavater II., hinsichtlich seines Umgangs mit Nachbildern allerdings überbietet er sein Vorbild. Denn seine vermeintliche 'Empathier gilt auch Unbelebtem: Mit seiner "Seele nun fuhr er in alles hinein; doch aber in Puppen vorzüglich" $(\mathrm{I} / 6,585)$. Aufgrund ihrer äußeren Gestalt behandelt er die Puppen so, als ob sie menschliche Seelen hätten, wobei ihn vor allem die zerstörte Integrität ihrer Körper schmerzt. Nachdem seine Schwester etwa in das "Wachslärvchen" einer alten "Puppe mit der Schere" eingestochen hatte, vergilt er's, indem er die Schwester ähnlich traktiert $(\mathrm{I} / 6,586)$. Da Nikolaus so weniger zur Beförderung der Menschen- als vielmehr der Puppenliebe physiognomiert, führt er die sittliche Dimension der Physiognomik ad absurdum (vgl. I/6, 665f.). Jean Pauk legitimiert Nikolaus' "Ärger« just mit der Gottebenbildlichkeit: "[D]ie Menschengestalt sei uns bis in jeden fernsten Nachschatten heilig ", da in das menschliche Gesicht gleichsam "Gottes Name« eingeschrieben sei $(\mathrm{I} / 6,586)$. Nikolaus' Puppenliebe setzt sich mit der Liebe zu einer "Kindheit-Puppe« $(\mathrm{I} / 6,627)$ wie zur geraubten Wachsbüste seiner angebeteten Amanda fort. Das der ätherischen Physiognomie Amandas ähnliche Wachsbild, das gestohlene "Gesicht der Prinzessin" (I/6, 639), bewahrt er nach seinem nächtlichen "Prinzessinraub« $(I / 6,625)$ in einer leeren Stutzuhr auf; es ist das passend gewählte Kästchen für die Verwahrung seines das Urbild repräsentierenden Uhrbilds (vgl. I/6, 637f.). Während die kindliche Puppenliebe das Abbild beseelt, wobei der innere Mensch als Puppe anschaulich wird, zeichnet sich die spätere »Ersatzmännin«-Liebe $(\mathrm{I} / 6,627)$ dadurch aus, dass die Wachsphysiognomie materialiter das innere Bild der Geliebten `im Kopf ‘ vorstellt. ${ }^{55}$ In Nikolaus' Physiognomik von Wachsbildern ist Lavaters Wissenschaft, die die Kluft zwischen den Subjekten zu überbrücken sucht, freilich um ihre sozialhermeneutische Dimension beschnitten. Ein intersubjektiver Sprung ist im Komet nur punktuell zwischen Nikolaus und Peter Worble möglich, wo der Vergleich der beiden »organischen Lesarten« ihrer Rippen $(\mathrm{I} / 6,598)$ ewige

54 Vgl. stellvertretend Klaas Huizing: Verschattete Epiphanie. Lavaters physiognomischer Gottesbeweis, in: Das Antlitz Gottes im Antlitz des Menschen. Zugänge zu Johann Caspar Lavater, hg. von Karl Pestalozzi und Horst Weigelt, Göttingen 1994, S. 61-78.

55

So schon Uwe Schweikert: Jean Pauls »Komet«, S. 54, zu den Amanda-Szenen S. 44-61. 
Freundschaft stiftet; ${ }^{56}$ er ist sonst - obgleich andersartig - dem Magnetismus/ Mesmerismus überantwortet. ${ }^{57}$ Physiognomik verweist immer nur auf das Subjekt zurück, das liest. Allerdings werden so die Möglichkeitsbedingungen des "Wahrnehmungs- und Verständigungsverhältnis« zwischen ego und alter offengelegt: ${ }^{58}$ Am Beispiel des Bephysiognomierens von Wachsbildern macht Jean Paul deutlich, dass der Mensch in intersubjektiven Verhältnissen generell auf seine Phantasie verwiesen ist.

Nicht nur Wachsbilder, sondern auch Gemälde stehen im späten Roman im Zeichen der Physiognomik. Auf seiner Reise kommt Hacencoppen in die Kunststadt Lukas-Stadt, in der sich zeitgleich zwei arme Malerschulen aufhalten, eine niederländische und eine italienische, die es auf das Geld des spendablen Fürsten abgesehen haben. Die beiden Schulen vertreten divergierende Programme der Gesichtsdarstellung, für die sich der Graf je ausspricht: Die Niederländer vertreten das Programm der »Natürlichkeit«, die Italiener das der »Verklärung« (I/6, 923). Hacencoppen, von dem 32 Porträts angefertigt werden, wird zu Repräsentationszwecken zum »Kunstmäzen« (I/6, 918; vgl. I/6, 935f.). Hauptzweck ist es aber, mit den Porträts den Vater und Amanda zu finden, die ihn anhand der gemalten Physiognomie erkennen sollen (vgl. I/6, 936, 939 und 957). Daher ist "gerade auf [s] ein Gesicht [s]eine ganze Zukunft und Krone gebaut« (I/6, 934).

Hacencoppens Ziel steht indes unter keinem guten Stern: Lavater wertet das Porträt bekanntlich gegenüber dem Schattenriß ab (vgl. F II, 91). »Portraetmahler« seien oft "Verlaeumder« (F II, 69), die die wahre Physiognomie nur unvollkommen darstellten (vgl. F II, 84). In der Tat handelt es sich bei den Luxstädter Malern lediglich um epigonale Kopisten, die gewissermaßen selbst Kopien sind. ${ }^{59}$ Von Hacencoppen treffen sie zwar alle etwas, aber doch mehr schlecht als recht (vgl. I/6, 957). Dabei wäre freilich auch zu fragen, welche Seele überhaupt herausphysiognomiert werden soll, wenn

56 Zur physiognomischen Konstruktion der Helden und der in der Physiognomie abgebildeten Freundschaft siehe Andreas Käuser: Die Verdopplung des Ich. Jean Pauls physiognomische Poetik im Komet, in: Jahrbuch der Jean-Paul-Gesellschaft 26/27 (1992), S. 183-196, zur Wachsbüste S. 185-193; vgl. zur Bildwerdung des inneren Menschen auch Stephan Pabst: Fiktionen des inneren Menschen. Die literarische Umwertung der Physiognomik bei Jean Paul und E.T.A. Hoffmann, Heidelberg 2007, S. 167-228, hier S. 193-196.

57 Siehe stellvertretend Maximilian Bergengruen: Pol und Gegenpol eines Magneten, S. 5360.

58 Andreas Käuser: Die Verdopplung des Ich, S. 190.

59 Siehe Monika Schmitz-Emans: Etüden über Plagiat und Fälschung. Ludwig Tiecks Novelle Die Gemälde und Jean Pauls Roman Der Komet, in: Die Prosa Ludwig Tiecks, hg. von Detlef Kremer, Bielefeld 2005, S. 115-135, bes. S. 133. 
Nikolaus seine eigne durch fremde ersetzt (vgl. I/6, 590). Hacencoppen sitzt beiden Schulen, wobei er je 16 Kopisten einer Schule "zugleich sitz[t], vorwärts und links und rechts, im Vollgesicht, im Profil, im Halbprofil, im Drittel-, im Viertelprofil« (I/6, 930).

Die Niederländer sind mit ihrem mimetischen Programm »echte[ ] Künstler«, denn "sie treffen jede Blatternarbe, jedes Nasenhaar« (I/6, 919). Sofern Hacencoppen in der Erzählerrede in einer »Viertelstunde [...] sechzehnköpfig" wird und dann "sechzehn Stirnen" und "sechszehn Nasen" (I/6, 939) bekommt, kann von einer inszenierten 'Anatomierung gesprochen werden, die an die Kopf-, Nasen- und Stirnstücke in Lavaters Fragmenten erinnert. In diesem Sinn resultieren aus den niederländischen Porträts zugleich Hacencoppens sphysiognomische Fragmente<, mit denen eine Dekomposition des Herrscherbildes einhergeht.

Die Fragmentierung wird in der Gesichtsmalerszene in der Bildlichkeit der (optischen) Farben weiter ausgefaltet. Die »Vervielfältigung des gräflichen Gesichts« (I/6, 921) vergleicht Jean Paul dabei mit dem »kleine[n] Dresdner Kirschkern ${ }^{60}{ }^{6}$ einer Miniatur, auf der 185 Gesichter eingeschnitzt sind: Hacencoppen bekommt "in einer Stunde mehr lange Nasen als ein anderer in seinem ganzen Dienste; denn sein Gesicht brach sich in den Wellen der Farben sechzehnmal« (I/6, 940). Sofern man die Gesichter wie auf dem Kirschkern kaum erkennen kann und sie zudem optisch gebrochen sind, steht freilich auch die physiognomische Durchsichtigkeit auf dem Spiel. ${ }^{61}$ Während Hacencoppen die Kunst der niederländischen »Kopisten« (I/6, 942) zunächst lobt, wertet er sie anlässlich seines Lobs der Italiener implizit wieder ab: Kunst sei »kein bloßes Silhouettenbrett des Gesichts oder eine englische Kopiermaschine der Gestalten, sondern eine selber gebärende Madonna« (I/6, 954). Damit wendet er sich mit Lavater gegen das "genau[e] [C]opieren « (F II, 82), zugleich aber auch gegen die von Lavater bevorzugten physiognomischen Medien. Die Italiener seien mit ihrem Programm der Verklärung "wahre[ ] Seelenmaler" (I/6, 920). Denn sie malen "das heilige ewige Innere« des Menschen »auf das Gesicht oder Porträt«, setzen dabei

60 Vgl. stellvertretend René von Niederhäusern: "So leserlich wie die Kehrseite der Gesetztafel«. Zur Bedeutung der Physiognomik in Jean Pauls Kampaner Tal, in: Physiognomie und Pathognomie. Zur literarischen Darstellung von Individualität, Festschrift für Karl Pestalozzi zum 65. Geburtstag, hg. von Wolfram Groddeck und Ulrich Stadler, Berlin/ New York 1994, S. 222-244.

61 Vgl. Mona Körte/Judith Elisabeth Weiss: Gesichter zwischen Erkennung und Auflösung. Eine Einleitung, in: Gesichtsauflösungen, hg. von dens., ZfL-Interjekte 4 (2013), S. 4-11, hier S. 10; Andreas Käuser: Die Verdopplung des Ich, S. 191. 
aber nicht auf herkömmliche Physiognomik, Mimik bzw. Pathognomik, da der Geist sich "nicht immer in Taten und Gesichtzügen" rege oder "sich doch nur in schlechten [...], in Farben, Mienen und Blicken" zeige (I/6, 954). Damit entwickelt Hacencoppen erneut ein ambivalentes physiognomisches Argument wider die Physiognomik. Da die Italiener sich am »Ideale einer Physiognomie« (I/6, 944) ausrichten, lassen sie nicht nur die Narben weg, sondern malen die Nase entsprechend dem klassischen Ideal. Hacencoppen sieht hier aus "wie ein Engel, man kannt' ihn kaum« (I/6, 955).

Nikolaus gefällt indes das Bild des kurzsichtigen Heiligenmalers, der schon »heilige Nikolause gemalt" hatte $(\mathrm{I} / 6,956)$, am besten. Damit erweist sich Nikolaus erneut als Lavater II., übersetzen doch die Maler bei Lavater die heilige Schrift des Gesichts (vgl. F II, 79). Auf dem Heiligenbild sieht sich Hacencoppen allerdings »kaum mehr gleich[] «; vielmehr findet er sich »dem Bilde ähnlich [...], das er sich selber in seiner Kindheit von seinem NamensHeiligen vorgemalt« $(I / 6,956)$. Bilder können demnach auch Vorbilder sein, an denen Identität allererst konstituiert wird: Wenn Hacencoppen gerade das unähnlichste Bild »für das schönste und ähnlichste» (I/6, 957) nimmt, dann auch, weil ihm im Heiligenbild materialiter das imaginäre Konstrukt seiner selbst entgegentritt. ${ }^{62} \mathrm{Da}$ Nikolaus in den welschen Porträts gar nicht mehr zu erkennen ist, verfehlen sie gleichermaßen das Herrscherbild wie die Physiognomie, anhand derer er erkannt werden sollte. Gemälde sind für Nikolaus insofern defizitär, als sie hinter seinem inneren Bild zurückstehen. Sofern die gemalten Porträts von Nikolaus' innerem Bild, d.h. von dem Gegenstand der Literatur, übertrumpft werden, artikuliert sich gerade an der Physiognomie eine literarische Bildkritik..$^{63}$

Dabei inszeniert Jean Paul mit der Gegenüberstellung von »Mimesis« und "Imagination« die Unterscheidung der poetischen Materialisten und Nihilisten aus der Vorschule. ${ }^{64}$ Die Gesichtsmalerszenen dienen dabei der Reflexion der Darstellung, die raffiniert die eigenen selbstreferentiellen Strukturen ins

62 Vgl. Vera Beyer und Jutta Voorhoeve: Nichts dahinter. Eine Einleitung, in: Das Bild ist der König, S. 7-12, hier S. 11.

63 Zur Bildkritik bei Jean Paul vgl. Monika Schmitz-Emans: Bildlichkeit, Bilder und bildende Kunst bei Jean Paul, S. 89 und 101; vgl. auch Alexander Honold/Ralf Simon: Vorwort, in: Das erzählende und das erzählte Bild, hg. von dens., München 2010, S. 9-24, hier S. 13: Poesie ist basal insofern als Bildkritik bestimmt, als »Textualität [...] das Bild [...] als ihr internes Anderes« entwirft.

64 Vgl. hierzu Monika Schmitz-Emans: Sankt Lukas als Statist. Malerfiguren und Malergeschichten bei Jean Paul, in: Jahrbuch der Jean-Paul-Gesellschaft 37 (2002), S. 53-85, bes. S. 63-71. 
Bild bannt (vgl. I/6, 940), wobei mit der Metaphorik des Malens für die Dichtkunst die Reflexion des eigenen Schreibens einhergeht.

Wo Jean Paul seinem Helden zutraut, idiosynkratisch Gemälde zu lesen, projiziert Nikolaus, obgleich er etwas anderes "vor der Nase hat« (I/6, 988), sich und seine Welt ins 'Bild $<$, indem er mit seiner physiognomierenden Phantasie ein X für ein U nimmt. In diesem Sinn stiftet Physiognomik als gestaltdeutende Kulturtechnik im Komet ein hermeneutisches Paradigma auch für die Interpretation von Alltagsszenen. Beispielsweise wird anlässlich der »Schreckenpost" (I/6, 964), der Ledermensch Kain - Nikolaus' teuflisches Pendant - habe auf den Dächern vermeintlich Hacencoppens Portativ-Residenzstadt Nikolopel in Brand stecken wollen, eine sSzene fiktionsintern Gegenstand verschiedener Auslegungen dessen, was es zu sehen gab. Deutlich wird dabei, dass die Lektüre der Welt ${ }^{65}$ aufgrund von Uneindeutigkeiten und falsch angewandtem Kontextwissen missglücken kann, ${ }^{66}$ wobei mit verschiedenen plots eines Geschehens das physiognomische Entzifferungsszenario gleichermaßen zum Schauplatz metanarrativer Reflexion wie eines apokalyptischen Zeichenchaos gerät. Hatte Lavater auf den undurchsichtigen Zeichenwald der Moderne mit einer sunschuldigen Semiotik und einer eschatologisch ausgerichteten Physiognomik geantwortet, so ist die physiognomische Heuristik in der Textwelt des Komet zwar als Orientierungsversuch ernst zu nehmen; das Scheitern der Deutungen an der Maskenhaftigkeit der Welt (vgl. I/6, 646) aber wird zugleich als Effekt einer nicht erlösungsfähigen Welt denkbar.

\section{Schluss}

Jean Paul codiert seinen Text mit dem Titel hinsichtlich eines Programms physiognomischen Entzifferns, das im Roman ausgefaltet wird. Die narbige Nase von Marggraf wird zum generischen Körperzeichen und unter je verschiedenen epistemischen Rahmen Vorzeichen der Heiligkeit oder vermeintlicher Garant für Kapital. Jean Paul reflektiert neben dem Restaurationskatholizismus und der Durchdringung modernen Lebens durch die Finanzökonomie mit seiner literarischen Physiognomik auch satirisch den gouvernementalen Zugriff auf das Subjekt. So erweist er sich mit seinem kritischen Rückgriff auf die widersprüchliche Physiognomik Lavaters

65 Vgl. Hans Blumenberg: Die Lesbarkeit der Welt, Frankfurt a.M. 1986, bes. S. 199-213.

66 Vgl. Umberto Eco: Lector in fabula. Die Mitarbeit der Interpretation in erzählenden Texten, übers. von Heinz G. Held, 3. Aufl., München 1998, S. 98-101. 
als scharfsichtiger 'Kulturphysiognom` von Modernisierungsprozessen des frühen 19. Jahrhunderts. Am Umgang mit Bildern werden dabei die Möglichkeitsbedingungen von Intersubjektivität offengelegt. Der Physiognomik wird nicht mehr zugetraut, die Kluft zwischen den Subjekten wechselseitig zu überbrücken; vielmehr wird Fremdverstehen überhaupt als phantastische Farçe gezeichnet. In einer Welt, in der die leere Parade die Hauptsache ist, prallen physiognomische Lektüren nicht nur an den trügerischen Oberflächen ab, sondern sie richten sich auch selbst auf den Schein aus. So wird Physiognomik unter dem Vorzeichen einer ungezügelten Phantasie, die Trost um den Preis der Narrheit verheißt, prekär.

Dabei nimmt Jean Paul die Physiognomik durchgehend zur Reflexion seines Textes in Dienst - von der Ebene des `Genres ‘ bis zur Ebene der Buchstaben: Mit der Inszenierung von Zeichentypen, Signifikation und genuin literarischen Zeichen dient Physiognomik der Reflexion von Semiologie und Fiktionalität. Wo sie Vehikel eines Bilddiskurses ist, werden mit ihr gleichermaßen die Darstellung, das Erzählen wie die Medialität des Textes bedacht. Dieses Fazit sei durch eine letzte Wendung nuanciert: Mit den sphysiognomischen Fragmenten wird textintern eine epistemologisch verunsicherte Perspektive des modernen Subjekts reflektiert, auf die Lavaters Physiognomik mit dem richtigen Blick und einem statischen Modell der Bedeutungsgeneration geantwortet hatte. Jean Pauls Textmodell dynamischer Bedeutungsträger steht dazu freilich quer. Sein Opus Magnum macht durch die Zirkulation sich spiegelnder Text-, 'Bildk- und Buchstaben-Partikel - der Hermetik eingedenk - zukunftsweisend Literatur zum Hort von Ähnlichkeiten. 